\title{
A game proposal to develop Computational Thinking and environmental awareness
}

Uma proposta de jogo para desenvolvimento do Pensamento Computacional e da consciência ambiental

\section{Fernanda Pires ${ }^{1,2}$, Fabrizio F. Honda, Guibson Silva, Rafaela Melo', Rosiane de Freitas $^{2}$, Marcela Pessoa ${ }^{1,2}$}

${ }^{1}$ ThinkTed Lab - Escola Superior de Tecnologia - Universidade do Estado do Amazonas (EST/UEA), Manaus-AM-Brasil.

${ }^{2}$ Instituto de Computação - Universidade Federal do Amazonas (PPGI/IComp/UFAM), Manaus-AM-Brasil.

\section{ARTICLE INFO}

Article history:

Received 03 March 2019

Accepted 07 April 2020

Available online 05 May 2020

Keywords:

Games for programming

Computational thinking

Programming Learning

ISSN: 2595-9077

DOI: JCThink.v3.n1.p111

\section{ABSTRACT}

INTRODUCTION: Discussions around the development of Computational Thinking have demanded efforts to propose methods, techniques and tools that can assist in this process, especially in the school context. This paper deals with the development of an educational game with the theme "Environment and Sustainability" involving the pillars of Computational Thinking. OBJECTIVE: The game aims to assist the development of Computational Thinking by solving the problems to be solved in the missions of the heroine Sofia. The proposal for creating the game points out ways to create tools that work across curriculum themes. Tactics usually incorporated into the algorithmic language that permeate the problem solving processes in Computer Science be used. METHOD: The creation of the game followed a development process based on agile methodologies. The human cognitive structure was considered together with the intertwining between the games and the four pillars of Computational Thinking for the composition of an architecture applied to the game map. RESULTS: The evaluation of the game map regarding the "algorithmization" of the phases fulfilled the requirements regarding the location of the pillars (Decomposition, Pattern, Abstraction and Algorithm). The game, as an entertainment product, was evaluated using the E-Game Flow which pointed to acceptance by the evaluators (undergraduate students in the Computing area) and some suggestions for improvement were recorded. CONCLUSION: Games are presented as an important tool regarding the development process of Computational Thinking considering the engagement they can cause in their users and the possibilities of using their mechanics for directed learning.

\section{RESUMO}

INTRODUÇÃO: As discussões em torno do desenvolvimento do Pensamento Computacional, têm demandado esforços no sentido de propor métodos, técnicas e ferramentas que possam auxiliar nesse processo, sobretudo em contexto escolar. $O$ presente artigo trata do desenvolvimento de um jogo educacional com o tema "Ambiente e Sustentabilidade" envolvendo os pilares do Pensamento Computacional. OBJETIVO: O jogo tem como objetivo auxiliar no desenvolvimento do Pensamento Computacional através da resolução dos problemas a serem resolvidos nas missões da heroína Sofia. A proposta de criação do jogo aponta formas de criar ferramentas que trabalhem temas curriculares de forma transversal. São usadas táticas usualmente incorporadas à linguagem algorítmica que permeiam os processos de resolução de problemas em Ciência da Computação. MÉTODO: A criação do jogo seguiu um processo de desenvolvimento baseado em metodologias ágeis. Foi considerada a estrutura cognitiva humana juntamente com o entrelaçamento entre os jogos e os quatro pilares do Pensamento Computacional para a composição de uma arquitetura aplicada ao mapa do jogo. RESULTADOS: A avaliação do mapa do jogo no que tange a "algoritmização" das fases cumpriu os requisitos quanto à localização dos pilares (Decomposição, Padrão, Abstração e Algoritmo). O jogo, enquanto produto de entretenimento, foi avaliado usando o E-Game Flow que apontou para aceitação dos avaliadores (alunos de graduação da área de Computação) e foram registradas algumas sugestões de melhoria. CONCLUSÃO: Os jogos se apresentam como uma importante ferramenta no que tange o processo de desenvolvimento do Pensamento Computacional considerando o engajamento que podem causar em seus usuários e as possibilidades de utilização de suas mecânicas para aprendizagem direcionada. 


\section{Introdução}

Muito vem se debatendo sobre o desenvolvimento do Pensamento Computacional (PC) como habilidade essencial para a resolução de problemas do cotidiano e sobre a forma de enxergar os elementos importantes de decisão (variáveis), bem como, a maneira de estabelecer conexões entre eles. Tais discussões ganharam forma através do discurso de Jeannette Wing (2006) que define PC como uma habilidade fundamental para qualquer pessoa e não somente um cientista da computação. A pesquisadora, destaca que o pensamento crítico deve ser considerado no processo de resolução de problemas, assim como as arquiteturas que fazem uso da computação. Além disso, afirma que o Pensamento Computacional é uma ferramenta para formalizar a resolução de problemas.

As estratégias de resolução de problemas são requeridas em todos os campos da vida, seja na execução de tarefas simples do dia a dia, como o percurso até a escola, ou em criar um algoritmo de criptografia de dados para a bolsa de valores. Alguns problemas são reconhecidamente de resolução difícil, isso está relacionado, entre outras coisas, ao conjunto de variáveis e processos associados. Poucos divergiriam quanto a complexidade de analisar o clássico problema da parada na Computação (Christian \& Griffiths, 2016), ou o número de conexões neurais possíveis em um humano adulto (Dehaene, 2014) ou, ainda, a modelagem matemática da Teoria dos Jogos (Sutiono, Purwarianti, \& Iida, 2014). Dificilmente alguém que não tivesse experiência em uma dessas áreas se disporia a traçar estratégias para a resolução dos problemas apresentados. Talvez houvesse desconhecimento em relação ao termo utilizado para descrever tais problemas, embora fosse possível reconhecer os passos estruturais traçados implicitamente para a solução deles.

Entretanto existe uma conjuntura em que essas estratégias para resolução de problemas ficam bem explicitadas. Este contexto é conhecido como jogos e acompanha a sociedade ao longo dos anos, desde os mais antigos, que tinham como cenário o coliseu romano, passando pelos tradicionais tabuleiros de xadrez e monopólio até os grandes títulos que tomam a cena através de consoles e computadores. Diferente de outros campos, traçar estratégias, fazer planejamento, eleger variáveis, escolher o menor caminho e criar planos de contingência é sempre divertido nos jogos. Partidas podem durar horas, dias e até meses, com direito a reuniões de planejamento criação de grupos entre outras tarefas que em outro cenário podem ser consideradas "enfadonhas".

Mais recentemente, os jogos de forma geral e estratégias gamificadas vêm sendo defendidos como alternativas lúdicas que podem ser utilizadas no processo de aprendizagem (Dichev \& Dicheva, 2017; Hamari et al., 2016; Plass, Homer, \& Kinzer, 2015; Qian \& Clark, 2016), por proporcionarem interação, imersão e consequente engajamento advindo do envolvimento entre o jogador e a jornada em execução (Klopfer, Haas, Osterweil, \& Rosenheck, 2018). A utilização de jogos em processos educacionais ainda suscita muitas discussões, pois criar um jogo com propósitos educativos exige mais do que o necessário a jogos comerciais de entretenimento, que, ainda assim, possuem um processo complexo de desenvolvimento.

O fato de viver em um "espaço" não necessariamente quer dizer que se consiga entender todas as relações que isso implica, esta é uma das razões para que o tema ambiente e sustentabilidade seja complexo e importante atualmente. A conscientização sobre os cuidados com o ambiente não tem se mostrado uma tarefa fácil, como evidenciam as discussões que acompanham os grandes tratados mundiais em nome do clima, entre elas as conferências definidas a partir do Clube de Roma (Fogel, 1994; Meadows \& Randers, 2012).

Se por um lado o desenvolvimento do Pensamento Computacional é essencial para a resolução de problemas, por outro atingir a sustentabilidade é um fator muito desejado, 
sobretudo no que tange ao tratamento do lixo e aos cuidados que podem ser realizados pela população. Considerando este contexto, propõe-se o desenvolvimento de um jogo eletrônico de propósito sério, aqui chamado de EcoLogic, em estilo plataforma que usa como base os quatro pilares do Pensamento Computacional na construção de estratégias para a resolução de problemas explicitados no gameplay.

O EcoLogic estabelece uma relação entre estratégias lógicas através de percursos em labirintos e a coleta seletiva de lixo. A proposta de criação do jogo visa responder como elementos importantes para o desenvolvimento do Pensamento Computacional podem ser adequados (planejados e mapeados) aos componentes curriculares, através de táticas usualmente incorporadas à linguagem algorítmica que permeia os processos de resolução de problemas em ciência da computação.

O restante deste artigo está organizado como segue. Na Seção 2 são apresentados os trabalhos relacionados. Na Seção 3 são apresentados os fundamentos teóricos sobre Pensamento Computacional, Jogos, Processos Educacionais e Ambiente e Sustentabilidade. Na Seção 4 é apresentado o jogo educacional proposto, EcoLogic, destacando a utilização dos pilares do Pensamento Computacional. Na Seção 5 são apresentados os resultados e discussões destes e na Seção 6 são feitas as considerações finais e indicados os trabalhos futuros.

\section{Trabalhos Relacionados}

Muitas discussões vêm sendo realizadas sobre a importância de levar o tema Pensamento Computacional para a escola de forma transversal e com isso trabalhar os temas curriculares. Os jogos têm se apresentado como ferramentas que podem ser usadas nesse processo. O jogo Robô Euroi se propõe a exercitar raciocínio lógico matemático e promover o desenvolvimento do Pensamento Computacional através de estruturas de decisão embutidas em uma aventura (Melo, Silva, Duarte, \& Pires, 2018).

No trabalho de Pessoa et al. (2019) é relatado um jogo cujo objetivo é a promoção do raciocínio viso-espacial através da busca por animais e é solicitado que o usuário crie e execute um algoritmo de forma intuitiva. A gamificação também ganha espaço diante da necessidade de novas abordagens para a educação no século XXI, no trabalho de Pires et al. (2019) é descrito um trabalho de gamificação para o desenvolvimento do Pensamento Computacional através de aprendizagem Matemática de forma lúdica que apresentou bons resultados no que diz respeito à aprendizagem e ao engajamento.

A sustentabilidade engloba conceitos interdisciplinares (ecologia, economia, cultura) além de estruturas sociais (indivíduos, famílias e comunidade). A compreensão desses ecossistemas é de extrema importância na formação do sentido de sociedade, comunidade e cidadania. O trabalho de Faria et al. (2019) apresenta o desenvolvimento de um jogo indígena Sateré Mawé cujo nome e identidade foram definidos conforme a importância atribuída à terra e à floresta para esses povos que apontaram que as novas tecnologias podem auxiliar em muitas coisas, inclusive na comunicação indígena.

Buscam-se formas de trabalhar conceitos relevantes, promover a conscientização sobre o ambiente e encontrar maneiras sustentáveis de habitar o mundo. Neste sentido, Santos, Strada e Bottino (2018), apresentaram uma proposta para promover essa aprendizagem através da utilização de jogos educacionais, que melhore o engajamento de forma lúdica, considerando os requisitos de aprendizagem e fatores de desenvolvimento humano.

Atualmente existe uma necessidade crítica de conscientização ambiental, a exploração de recursos naturais em excesso ainda é um problema atual, por isso, a importância emergencial de adotar práticas de desenvolvimento sustentável. Segundo Katsaliaki e Mustafee (2012), uma maneira de promover a consciência ambiental para um 
futuro mais sustentável é por meio de jogos sérios voltados para a prática da sustentabilidade.

Wang \& Tseng (2014) consideram a educação ambiental emergente e relevante para a educação do ser humano, além disso, acreditam que a junção de tecnologia e consciência ambiental é concebível, porém, não é uma tarefa simples. Em seu estudo, os autores aplicaram um jogo sério para a educação ambiental de autoria própria. Por acreditar que os jogos sérios tornam a aprendizagem algo envolvente, Hedin et al. (2017) criaram um jogo para ajudar as pessoas a se conscientizar sobre seu próprio consumo de energia, direcionando-as a uma mudança comportamental voltada para a sustentabilidade.

Qian e Clark (2016) apresentam uma revisão sistemática da literatura sobre aprendizagem baseada em jogos (Game-Based Learning GBL), bem como teorias de aprendizagem e habilidades do Século XXI. Segundo a revisão da literatura realizada por eles, GBL pode ser eficaz no que tange a simplificação do processo de desenvolvimento dessas habilidades e, frente a isto, é mostrado o exemplo do atual currículo escolar de Hong Kong, que enfatiza a importância da construção da criatividade dos alunos, e assim, os professores são incentivados a promover inovação.

Diferente das pesquisas anteriores, o presente trabalho integra a importância da sustentabilidade e do Pensamento Computacional através do potencial apresentado pela aprendizagem baseada em jogos. O serious game intitulado EcoLogic, possui temática de meio ambiente e instiga a resolução de problemas a partir de estratégias baseadas nos quatro pilares do Pensamento Computacional.

\section{Fundamentos Teóricos}

Ao propor a utilização dos quatro pilares do Pensamento Computacional para projetar um jogo de propósito sério, cujo tema é ambiente e sustentabilidade, se faz necessário descrever os aspectos mais importante para o trabalho. Neste sentido, esta seção descreve sobre Pensamento Computacional, Jogos, Processos Educacionais e Ambiente e Sustentabilidade.

\subsection{Pensamento Computacional}

Wing (2006) considera o Pensamento Computacional (PC) como uma habilidade fundamental para todos os seres humanos, que permite a resolução de problemas de forma analítica e diz respeito a como as pessoas veem e estruturam o pensamento. Dessa forma, o assim como a leitura, a escrita e a aritmética têm espaço nos currículos escolares, desenvolver o Pensamento Computacional precisa ganhar relevância uma vez que também contribui para fortalecer a capacidade analítica das pessoas, especialmente se explorado desde a infância. Alguns países como Austrália, Reino Unido e EUA vêm demonstrando preocupação com o processo de desenvolvimento do PC nas crianças, uma inquietação que começou com a preocupação de adicionar computação/informática ao currículo escolar.

Em 2010, Smith, Sutcliffe e Sandvik (Smith, Sutcliffe, \& Sandvik, 2014), observaram que nas escolas do Reino Unido o currículo das Tecnologias da Informação e Comunicação (TIC) era realizado de maneira a perpetuar a função de usuário de tecnologia e não criador. Propuseram, então, a criação de clubes de programação. Observaram que os processos de pensamento utilizados na resolução de um problema, cuja representação permite a execução por um agente de processamento de informações, poderia ser uma ferramenta poderosa no desenvolvimento do raciocínio lógico. PC diz respeito a forma como as pessoas pensam e organizam as informações de maneira a criar inferências sobre o mundo. Essa estruturação pode ser ou não otimizada no que tange o 
tempo de resposta, o custo envolvido além do impacto na resolução do problema analisado.

Pensamento Computacional é um conjunto de habilidades que permite a resolução de problemas. A proposta da BBC (2018) elegeu conceitos amplamente utilizados em Ciência da Computação na composição de estratégias para a resolução de problemas e estruturou os quatro pilares fundamentais: i) Decomposição, ii) Reconhecimento de Padrões, iii) Abstração e iv) Algoritmos. Ao se deparar com uma tarefa difícil, "pensar computacionalmente" é reestruturar o problema de forma que a solução seja mais facilmente encontrada, fazendo um paralelo com a forma que as máquinas chegam a soluções de problemas complexos. A Figura 1 apresenta a conexão entre os quatro pilares que sustentam o Pensamento Computacional e está melhor explicada a seguir.

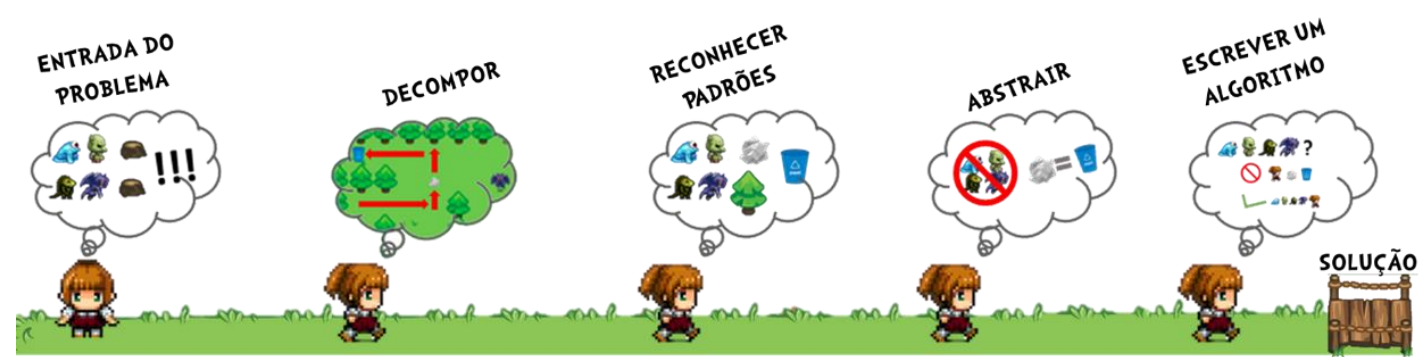

Figura 1. Processo de resolução de um problema, segundo os pilares do Pensamento Computacional.

A primeira etapa é a Decomposição. Ao receber um problema complexo, o indivíduo deve dividi-lo em partes menores para facilitar a compreensão e otimizar o entendimento. Após decompor o problema, a segunda fase é o Reconhecimento de Padrões. Os problemas menores são examinados de forma a encontrar padrões entre eles. Tais padrões são úteis, pois, ao encontrar a solução para um dos problemas pequenos, pode-se reutilizá-la para a resolução de outros problemas, ou seja, a solução pode ser replicável. Quanto mais padrões foram encontrados, mais fácil e rápida se torna a resolução do problema.

A terceira etapa do processo é a Abstração. Talvez este seja um dos mais importantes pilares. Nele são identificadas as características mais relevantes para solucionar o problema, desconsiderando detalhes de menor relevância, e, desta forma, a ideia geral da solução é formalizada (BBC, 2018). A quarta e última etapa é a formalização dos algoritmos capazes de serem aplicados com a finalidade de resolver o problema. Após decompor, reconhecer padrões e abstrair, são determinadas regras para a resolução dos problemas. Um algoritmo é um conjunto de instruções que descreve os passos para solucionar um problema (Cormen, Leiserson, Rivest, \& Stein, 2001).

Existem discussões sobre os processos de aprendizagem humanos e sobre a alta valorização do termo "ensinar" (Resnick \& Robinson, 2017). Diante dos modelos que veem o indivíduo como protagonista de seu processo de aprendizagem (Christian \& Griffiths, 2016; Mayer, 2015; Resnick \& Robinson, 2017), defende-se aqui que o termo "Ensino de Pensamento Computacional" é contraditório. Acredita-se que não é possível ensinar a pensar, especialmente pelo que preconizam os estudos sobre o conceito de PC, enquanto conjunto de habilidades cognitivas que permitem solucionar problemas e modelar soluções que serão computáveis por máquinas (computadores). Sendo assim, pode-se inferir que o PC é inato e inerente a estrutura biológica humana, que pode ser desenvolvida ao longo da vida de acordo com o estímulos internos e externos (Pires et al., 2018). 


\subsection{Jogos e processos educacionais}

A humanidade passou por um longo processo evolutivo e antes mesmo da invenção da escrita já havia métodos de aprendizagem que sobreveio gerações. As buscas de soluções para os problemas apresentados promoveram o avanço dos primórdios da Ciência e da Tecnologia. Huizinga (2014) afirma que os jogos mesmo antecedendo a cultura, se apresentam como fator constituinte de um processo cultural e contribuem para a composição do conhecimento.

O conceito de jogo passou por mudanças ao longo do tempo. É possível observar uma influência das visões sociais de cada época. Wittgenstein (1953) ao tratar de jogos da linguagem concluiu que os conceitos, regras e competições não eram usados de forma correta, incorrendo assim na definição e uma série de comportamentos humanos que não poderiam ser definidos como jogos. Roger Caillois (2001) na década de 1960 definiu os jogos como uma atividade voluntária, separada em tempo e espaço, regida por regras e que te faz acreditar nos acontecimentos envolvidos e essenciais ao processo de desenvolvimento humano. Suits (1978) define que para jogar é necessário se engajar em uma atividade específica usando o que é permitido pelas regras e estas são responsáveis por tornar cada atividade possível. Para Crawford (1981) jogos são a junção de quatro fatores: representação (modelos formais da realidade), interação, conflito e segurança.

Katie Salen e Eric Zimmerman (2003) definem jogos como sistemas cujos jogadores estão engajados em conflito artificial. É definido pelas regras e o resultado é quantificável. Scott Rogers (2014), em uma tentativa lúdica, define jogo como uma atividade que requer no mínimo um jogador, possui um sistema de regras e uma condição de vitória. Pela evolução dos conceitos é possível avaliar o estado de desenvolvimento da linha de estudos bem como sua área de aplicação. O sucesso de um jogo vem sendo ligado às características humanas biológicas no campo da neurociência, o que não seria possível anteriormente pela falta de pesquisas científicas nessa área (Gee, 2003).

Na década de 1970, começam algumas discussões sobre como os jogos poderiam ser usados especificamente para aprendizagem (Abt, 1987). São chamados de Serious Game e ao longo do tempo ganharam outros nomes como: jogos educativos, jogos educacionais, jogos de propósito sério, resonant games e até mesmo brain games. O jogo mantém as pessoas engajadas no cumprimento de uma missão, são estabelecidas estratégias, exploram possibilidades e o jogador é o protagonista do enredo. Tais características são consideradas importantes no processo de aprendizagem.

Ao analisar a importância da disseminação de métodos e técnicas capazes de auxiliar no desenvolvimento do Pensamento Computacional, observa-se a utilização de jogos digitais no cenário educacional e como tem se apresentado como uma ferramenta com vistas a tornar a aprendizagem mais atraente e divertida (Savi \& Ulbricht, 2008). Sabe-se que as crianças de hoje nascem em meio a tecnologias digitais, crescem utilizando jogos de computador, videogames e smartphones, reservando grande parcela do tempo a estas atividades. Assim, os "estímulos" hoje são diferentes de 30 anos atrás. Conforme a neuroplasticidade, o cérebro tem a capacidade de criar novas conexões com base nas entradas recebidos, orquestrando, com isso, o processo de aprendizagem. Considerando os fatores biológicos e a familiaridade com as mecânicas de jogos, surgem propostas de aplicação destes no cenário educacional, ou ainda a gamificação de processos com mesmo cunho (Dichev \& Dicheva, 2017).

Para que a aprendizagem por meio dos jogos seja efetiva é preciso considerar os aspectos que promovem a diversão, como nos jogos comerciais e os aspectos cognitivos humanos, ou seja, no processo de desenvolvimento de um jogo educacional é importante pensar que habilidades e estratégias resultarão no aprimoramento de capacidades intelectuais (Gros, 2007). Geralmente, os jogos comerciais são projetados para 
proporcionar diversão sem ênfase no processo de aquisição de conhecimento, embora Gee (2003) afirme que, mesmo indiretamente, se aprende através de jogos motivados pelas emoções e conexões neurais envolvidas pela imersão e outros fatores.

A constituição de um jogo educacional predispõe uma série de fatores que precisam ser pautados e avaliados para serem implementados. A composição de um jogo educacional que de fato consiga atender aos requisitos lúdicos sendo educacionalmente construtivo é um desafio (Berg Marklund, 2013). Para tanto, a arquitetura precisa estar de acordo com as estruturas cognitivas humanas e respeitando os limites das cargas cognitivas de acordo com a Teoria Cognitiva da Aprendizagem Multimídia (Mayer, 2015).

Quando implementados considerando os requisitos de aprendizagem, os softwares educacionais possuem diversos benefícios, alguns deles são (Hsiao, 2007; Mitchell \& Savill-Smith, 2004):

- Efeito motivador: por serem atraentes e divertidos, os jogos costumam deixar o jogador mais relaxado, proporcionando maior disposição para o aprendizado (Hsiao, 2007). Os frequentes desafios despertam nas pessoas motivação para vencê-los;

- Aprendizagem facilitada: conteúdos considerados difíceis podem ser representados de maneira mais simples no cenário de um jogo, facilitando dessa forma, o aprendizado. Os jogos podem também estimular a aprendizagem por meio da tentativa e erro (Mitchell \& Savill-Smith, 2004);

- Desenvolvimento de habilidades cognitivas: ao longo de um jogo, o usuário é constantemente encorajado a desenvolver estratégias, aspecto relevante para a resolução de problemas, memorização, raciocínio dedutivo (McFarlane, Sparrowhawk, \& Heald, 2002), insight e desenvolvimento de habilidades analíticas e computacionais (Mitchell \& Savill-Smith, 2004).

Vale ressaltar que um importante papel desenvolvido pelos jogos é possibilitar a simulação do mundo real em ambientes digitais, permitindo vivenciar experiências sem de fato correr riscos, de forma engajadora em decorrência das mecânicas envolvidas.

\subsection{Ambiente e sustentabilidade}

O surgimento do termo sustentabilidade foi oficialmente introduzido no encontro internacional The World Conservation Strategy (Calvacanti, 1995), ao apresentar termo Desenvolvimento Sustentável em 1980, projetando o mundo ideal através de práticas capazes de satisfazer o presente sem influenciar ou comprometer o desenvolvimento das novas gerações (Siche, Agostinho, Ortega, \& Romeiro, 2007).

Apesar de que atualmente o termo Sustentabilidade é comumente associado a outros termos, Siche et al. (2007) refere-se à sustentabilidade como tudo que pode ser mantido, conservado ou suportado. Sachs (1989) descreve a sustentabilidade como um conceito dinâmico que toma como base as constantes necessidades advindas da sociedade. No contexto de sustentabilidade, encontra-se a educação ambiental, tendo como objetivo central auxiliar nas boas práticas que guiam a relação do homem com ambiente (Sauvé, 2005).

Em tempos em que as informações possuem um papel de extrema relevância para o contexto social, a educação ambiental juntamente com cidadania detém a grande capacidade de motivar e sensibilizar pessoas para transformar as diversas formas de participação da defesa da qualidade de vida (Jacobi, 2003). Deste modo, entende-se que a educação ambiental é condição necessária para que haja a uma modificação no quadro socioeconômico (Sauvé, 2005), por isso, este trabalho tem como objetivo a promoção de educação ambiental no que diz respeito a coleta seletiva através de um jogo eletrônico que, de forma implícita, promove o desenvolvimento de estruturas do Pensamento Computacional. 


\section{Desenvolvimento do Jogo}

EcoLogic é um jogo digital educacional de ação e estratégia em formato de puzzle, para plataformas móveis. Possui perspectiva top-down e envolve mecânicas de estilos de jogos de ação e aventura, além disso, possui personagens com estilos artísticos de RPG Maker. Procura introduzir noções de educação ambiental, relacionadas à coleta seletiva, através da associação de tipos de lixos às suas respectivas lixeiras e, com isso, disseminar o pensamento computacional voltado para a resolução de problemas lógicos. Esta seção descreve o cenário do jogo e as ferramentas utilizadas no processo de desenvolvimento, tendo como base o GDD (Game Document Design).

\subsection{Cenário e Fluxo do Jogo}

Sofia era uma garotinha que morava em um pequeno vilarejo na companhia de sua avó, Mônica, ao Sul da grande cidade. Sua rotina diária, além de seus afazeres escolares, era cuidar do jardim de sua casa. Um de seus maiores interesses era observar as árvores com suas amigas. Certo dia, ao sair da escola ela percebeu que a paisagem não era mais a mesma. O lago estava repleto de lixo, algumas árvores queimadas com galhos retorcidos e o céu estava nublado. Chegou em casa entristecida e sua vó foi conversar com ela. Mônica contou à neta que há muito tempo trabalhara em uma fábrica que estava projetando uma máquina para erradicar todo o lixo do planeta, mas, durante os experimentos algumas coisas saíram do controle e o lixo coletado havia ganhado vida. $\mathrm{Na}$ época os seres foram aprisionados e um antídoto, guardado em uma sala segura no subsolo, havia sido desenvolvido. Pelo que Mônica havia visto, alguém ativou a máquina e as criaturas precisavam ser paradas.

EcoLogic é sobre a jornada da heroína Sofia que parte para a fábrica, em que sua avó havia trabalhado, com o objetivo de salvar o planeta das criaturas de lixo. O jogo possui quatro fases: floresta, campo, cidade e fábrica. A Tabela 1 apresenta cada uma das fases relacionando respectivamente a quantidade de inimigos, lixeiras e lixos.

O tem arquitetura de cenários semelhantes e os objetivos têm seu nível de dificuldade aumentado à medida que progridem, cumprindo com a finalidade de auto regulação de aprendizagem associada às habilidades motoras necessárias. O público alvo são crianças na faixa etária entre 7 e 12 anos, alfabetizadas e com acesso a um celular com Sistema Operacional Android.

\begin{tabular}{lccc}
\hline \multicolumn{1}{c}{ Fase } & Qtd de inimigos & Qtd de lixeiras & Qtd de lixo \\
\hline Fase 1: Floresta & 5 & 5 & 5 \\
\hline Fase 2: Campo & 6 & 10 & 10 \\
\hline Fase 3: Cidade & 7 & 12 & 12 \\
\hline Fase 4: Fábrica & 8 & 16 & 16 \\
\hline
\end{tabular}

Tabela 1. Fases do jogo com seus respectivos elementos.

O objetivo em campo é colocar cada um dos lixos espalhados em suas respectivas lixeiras (papel, plástico, vidro e metal), ao mesmo tempo que se esquiva dos inimigos, conforme surgem, abrindo caminho para a próxima fase.

O mapeamento lógico de cada fase, criado pelo jogador, é essencial para alcançar a condição de vitória. Para cumprir as tarefas propostas pelo jogo, o usuário precisa usar raciocínio lógico. A tela de ação foi dividida em uma grande matriz onde os elementos estão dispostos como em um tabuleiro. Como obstáculos fixos, foram colocadas várias árvores, algumas em sequência de barras, outras em sequências de "L". As lixeiras estão dispostas de forma aleatória pelo cenário. Na primeira fase existe um único tipo de lixeira, 
e o dever do jogador é simplesmente não se deixar alcançar pelos "monstros" dispostos pelo cenário e colocar o lixo nas lixeiras disponíveis.

A cada toque em um dos monstros localizados em pontos estratégicos, uma porcentagem de vida é perdida (corações) se os três corações forem eliminados o jogo é encerrado. Um movimento impensado, pode levar o lixo para um canto em que não se pode retirar (peça morta), no estilo mecânico do Sokoban (puzzle que pode ser usado para emular um autômato limitado linear - Máquina de Turing de fita finita) ${ }^{1}$ (Culberson, 1997).

Para fazer o descarte correto dos itens de lixo, o jogador precisa movimentar a personagem através dos comandos touch screen nas extremidades da tela - direita, esquerda, superior e inferior - conforme apresentando na Figura 2. É a personagem que levará o material para as lixeiras específicas.

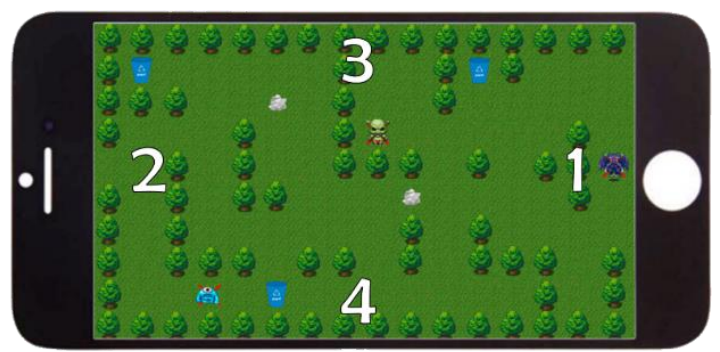

\begin{tabular}{|c|c|c|}
\hline $\mathbf{N}$ & Movimento & Sprite \\
\hline 1 & Move $\mathrm{p} /$ direita & \\
\hline 2 & Move $\mathrm{p}$ / esquerda & \\
\hline 3 & Move $\mathrm{p} /$ cima & \\
\hline 4 & Move p/ baixo & \\
\hline
\end{tabular}

Figura 2: Demonstração de movimentação na tela.

As telas iniciais do jogo, apresentadas na Figura 3, são, respectivamente, o menu principal e a tela de seleção de fases. As cores e os elementos que compõem as telas, foram escolhidos para remeter ao tema do jogo, que é o meio ambiente. A faixa etária do jogo também contribuiu para a escolha da composição do design.
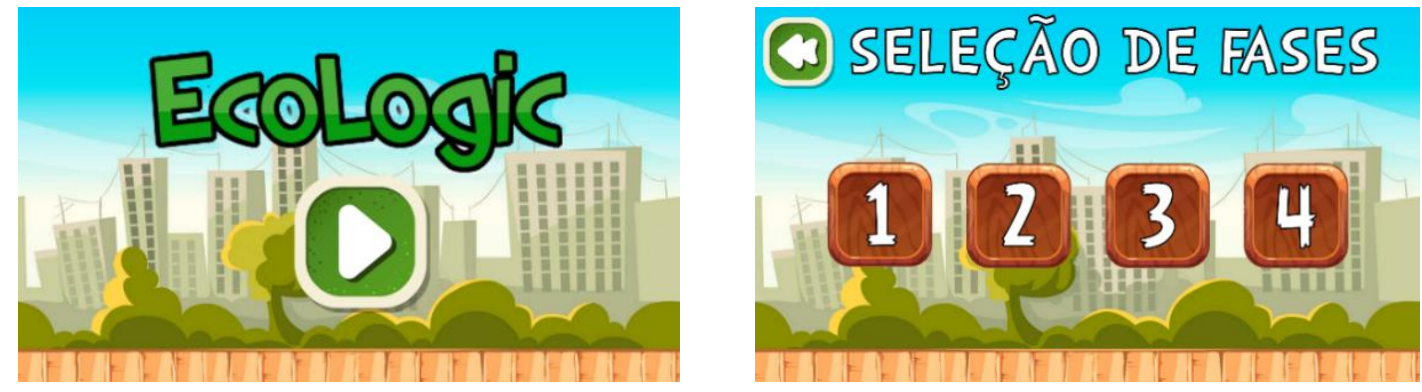

Figura 3. Tela Principal e Seleção de Fases.

Após clicar para jogar a primeira fase do jogo, o jogador é direcionado para as telas de storytelling (Figura 4). Nestas telas o jogador tem acesso à história geral do jogo.

${ }^{1}$ It is shown that the popular puzzle Sokoban can be used to emulate a linear bounded automata (finite tape Turing Machine (TM)). In particular, a construction is given that has a solution if and only if the corresponding Turing Machine on its input halts in the accept state. Further, if the TM halts and accepts, then the pusher will make $\theta(n+t(n))$ moves and pushes, where $n$ is the number of symbols on the input tape, and $t(n)$ is the number of transitions made by the TM during its computation. 

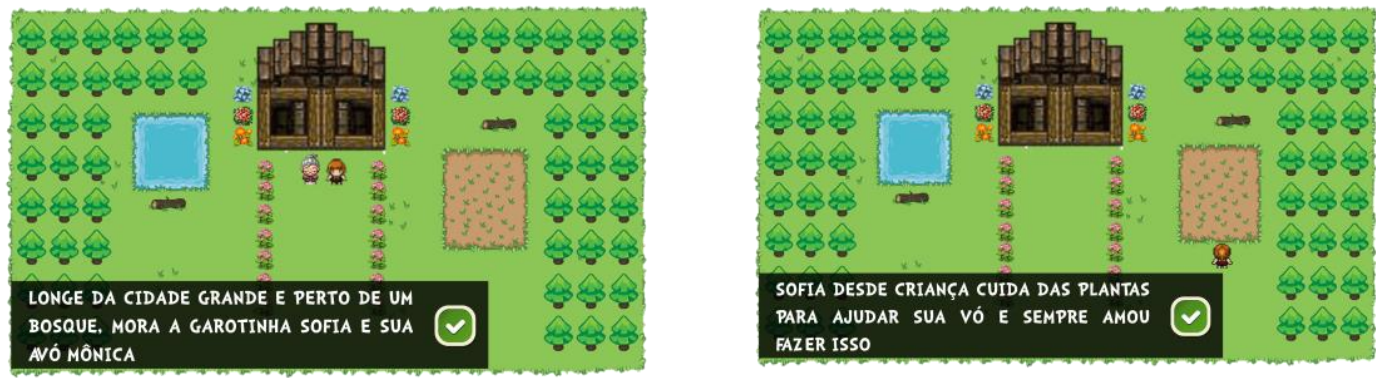

Figura 4: Telas de Storytelling em que Sofia recebe sua missão.

Ao longo de sua jornada, o usuário é sempre informado sobre o estado do jogo, por meio de feedback (telas de pop-up), como exemplificado na Figura 5.

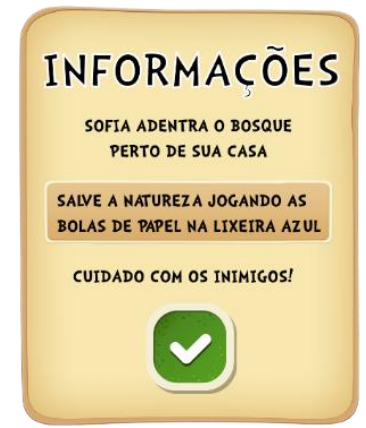

Figura 5: Pop up de orientação da primeira fase.

A visão top-down, Figura 6, e a movimentação da câmera que acompanha o jogador são algumas das características do EcoLogic. A vitória é condicionada a dois critérios: i) o jogador precisa desviar dos inimigos para não sofrer danos e "perder sua vida" e ii) é necessário fazer a coleta seletiva de todo material espalhado pelo cenário, só assim a porta que leva para o próximo nível é desbloqueada. As telas apresentadas na Figura 6 ilustram as fases um e dois do jogo. As fases são compostas por elementos que remetem à natureza, além dos objetos essenciais a um jogo: botões, Head Up Display (hud), personagem, inimigos e obstáculos.
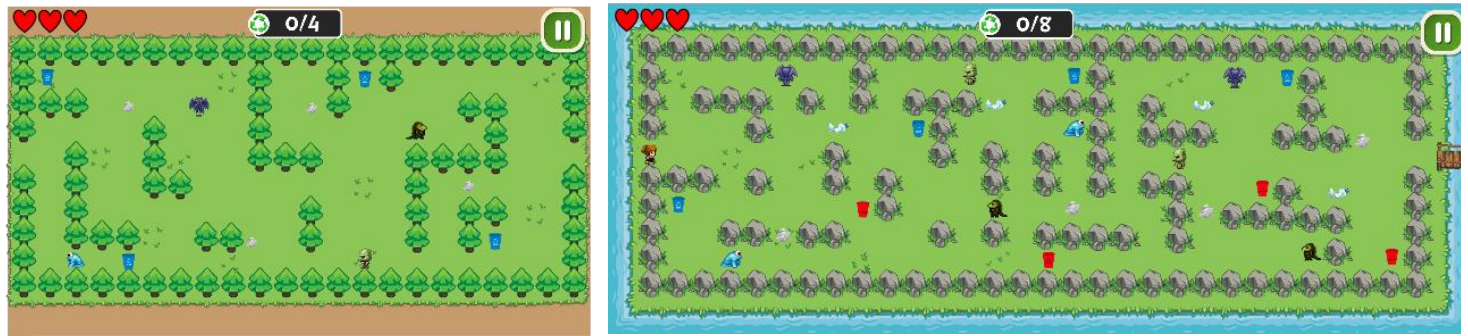

Figura 6: Fases do jogo.

A Tabela 3 apresenta a personagem principal e alguns dos inimigos, que assim como os outros elementos do jogo, estão disponíveis gratuitos na internet.

Com o objetivo de apresentar o processo existente no jogo, foi construído o diagrama de estado apresentado na Figura 7. É possível observar a mecânica de passagem de níveis, o processo de interação entre as fases do jogo bem como os artefatos envolvidos. 


\begin{tabular}{ll}
\hline Assets & Nomes/funções \\
\hline Sofia & $\begin{array}{l}\text { Personagens do jogo. Sofia a heroína e sua avó } \\
\text { Mônica (não jogável) }\end{array}$ \\
\hline Lixos disponíveis no cenário & $\begin{array}{l}\text { Vilões: Porcujo, Gosmento, Jacaréca, Gargulixo } \\
\text { jogações representam a quantidade de vidas do } \\
\text { coração é perdido }\end{array}$ \\
\hline
\end{tabular}

Tabela 3: Elementos do Jogo.

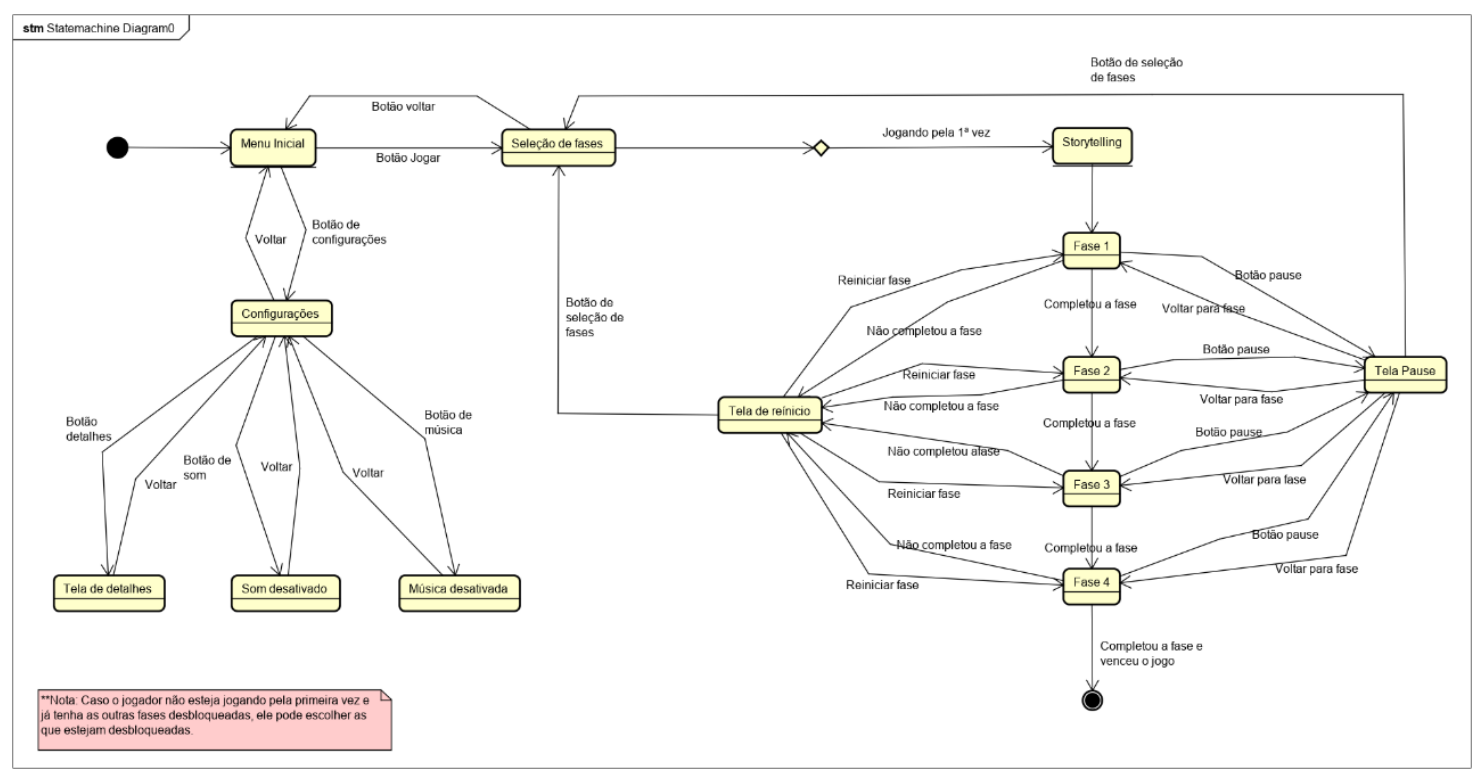

Figura 7: Diagrama de estados do EcoLogic.

\subsection{Ambiente de Desenvolvimento}

O processo de desenvolvimento da ferramenta foi dividido em etapas: Modelagem visual, que compreende o design visual e de interação e a codificação. Na primeira parte, a partir do mundo do jogo foram selecionados os elementos que deveriam compor o universo do EcoLogic. As imagens foram selecionadas separadamente de sites de livre acesso e organizadas através de processo de composição usando softwares de edição de imagem e vetorização. Esse processo resultou em um mockup cujos testes foram realizados de forma mecânica (sem uso de funções programadas).

$\mathrm{Na}$ segunda fase (decodificação) a game engine selecionada foi o Unity $2 \mathrm{~d}$, considerando o desempenho, os graus de liberdade quanto a licença (gratuita em nível educacional) e o histórico da ferramenta no que diz respeito à clareza, bibliotecas, e 
visualização das tarefas executadas. Além das possibilidades de exportação para os formatos Android, iOS, Windows e web (multiplataforma).

\subsection{Pilares do Pensamento Computacional aplicados ao EcoLogic}

Ao considerar o objetivo para desenvolver o jogo, algumas decisões de projeto foram tomadas. A forma que as pessoas aprendem (Mayer, 2015) e como se constroem subsunçores ao longo da vida (Mack, Schwartz, Jessell, Siegelbaum, \& Hudspeth, 2013), mediaram a escolha dos alicerces sobre os quais as estruturas para aprendizagem e promoção do desenvolvimento do Pensamento Computacional deveriam fundamentar-se, especialmente, em se tratando de jogos. Tais alicerces são storytelling, interface, mecânica e gameplay e são ilustrados no esquema apresentado na Figura 8, incluindo a conexão com os aspectos neurológicos até resultar na sistematização da arquitetura do jogo.

A partir da narrativa do jogo, foram definidas a missão e todos os elementos que deveriam compor o mundo do EcoLogic. A arquitetura do cenário (ordenação de obstáculos, vilões, passagem de nível) considerou os requisitos biológicos de percepção e processamento da informação (Mayer, 2015) em ambientes digitais bem como os puzzles foram projetados de maneira a apresentar determinadas situações problema.

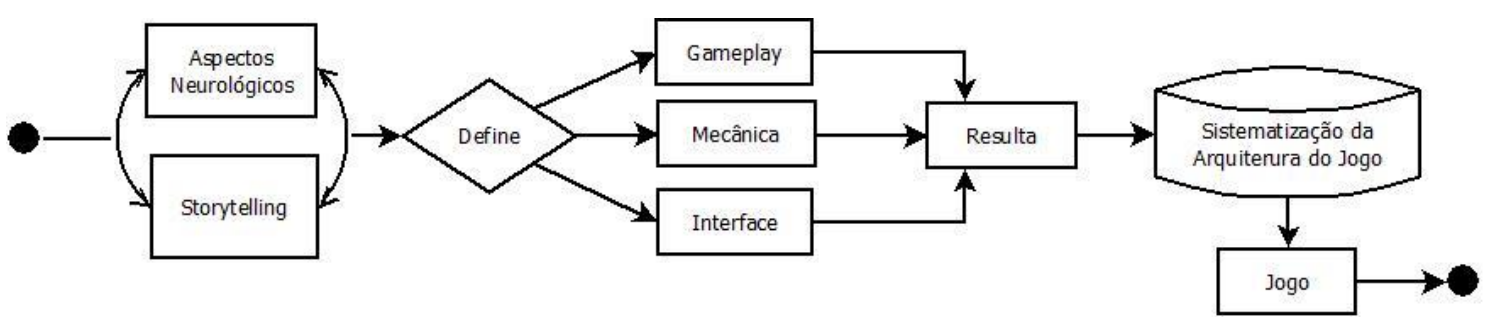

Figura 8: Esquema que ilustra como as estruturas para aprendizagem e promoção do desenvolvimento do PC são fundamentadas.

Um dos casos foi a implementação da dinâmica mecânica do Sokoban. Para esse modelo, o "lixo" foi considerado como elemento de atração (power-up), dado o contexto do jogo a inclinação do jogador deve ser a de capturá-lo. O grau de dificuldade foi atribuído usando os seguintes recursos (variáveis): i) a localização do power-up, ii) a presença ou não do vilão no quadrante e iii) o padrão de movimentação do mesmo. Dessa forma o usuário precisa tomar decisões sobre qual a melhor estratégia para atingir o objetivo. Em alguns quadrantes só é possível pegar o lixo se perder uma vida. Nesse caso o usuário precisa se manter ileso até chegar a este ponto, pois sem vida não alcançará a condição de vitória. Outra decisão é com relação a abordagem (padrão de movimentação) para capturar o lixo. Dependendo do lugar em que o mesmo está localizado, a "entrada" da personagem terá que ser diferente, senão aprisiona uma peça precisando reiniciar a trama. O Quadro 1 apresenta as fases do jogo e seus respectivos algoritmos evidenciando os pilares do Pensamento Computacional que são utilizados e as estratégias para percorrer o caminho que leva ao sucesso no jogo. 


\begin{tabular}{|c|c|}
\hline 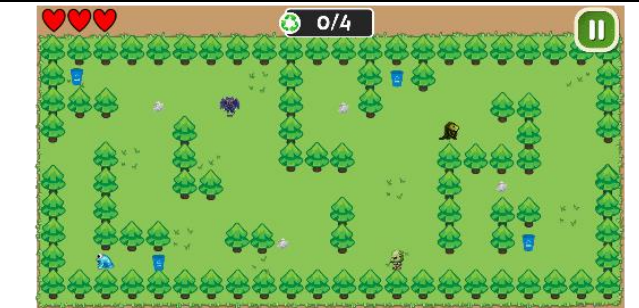 & 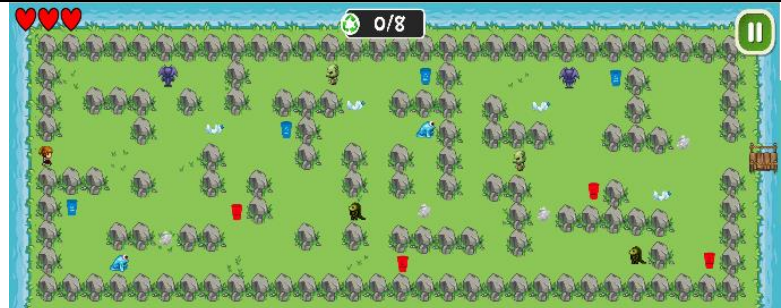 \\
\hline $\begin{array}{l}\text { Fase 1: } \\
\text { Inicio_algoritmo } \\
\text { Qtde_papel=4 } \\
\text { Qtde_vidas=3; } \\
\text { Enquanto (qtde_papel !=0) } \\
\text { Se "caminho_frente_livre" \& } \\
\text { "sem_inimigos" } \\
\text { mova_frente(); } \\
\text { Senão se "caminho_esquerda" \& } \\
\text { "sem_inimigos" } \\
\text { vire_esquerda(); } \\
\text { Senão se "caminho_direita" \& } \\
\text { "sem_inimigos" } \\
\text { vire_direita(); } \\
\text { se "tocando_em_inimigo" } \\
\text { qtde_vidas--; } \\
\text { se "papel_tocando_lixeira" } \\
\text { qtde_papel--; } \\
\text { Fim_algoritmo }\end{array}$ & $\begin{array}{l}\text { Fase 2: } \\
\text { Inicio_algoritmo } \\
\text { Qtde_papel=4; } \\
\text { Qtde_garrafas=4; } \\
\text { Qtde_vidas=3; } \\
\text { Enquanto (qtde_papel != } 0 \| \text { qtde_garrafas!=0) } \\
\text { Se "caminho_frente_livre" \& "sem_inimigos" } \\
\text { mova_frente(); } \\
\text { Senão se "caminho_esquerda" \& "sem_inimigos" } \\
\text { vire_esquerda(); } \\
\text { Senão se "caminho_direita" \& "sem_inimigos" } \\
\text { vire_direita(); } \\
\text { se "tocando_em_inimigo" } \\
\text { qtde_vidas--; } \\
\text { se "papel_tocando_lixeira_azul" } \\
\text { qtde_papel--; } \\
\text { se "garrafa_tocando_lixeira_vermelha" } \\
\text { qtde_garrafas--; }\end{array}$ \\
\hline
\end{tabular}

Quadro 1. Organização das fases e os algoritmos de resolução.

Considerando que o Pensamento Computacional tem seu suporte em quatro pilares: Decomposição, Reconhecimento de Padrão, Abstração e Algoritmos e que este trabalho busca evidenciar que tais pilares podem ser aplicados a outras áreas do conhecimento, a Tabela 4 estabelece uma relação entre os pilares do pensamento computacional, estruturas computacionais e a relação que se pretende com o meio ambiente. Todos estes fatores foram considerados no processo de composição do mapa lógico do jogo, em que os pilares do PC foram decompostos e diluídos no cenário do jogo.

\begin{tabular}{ll}
\hline $\begin{array}{c}\text { Pensamento } \\
\text { Computacional }\end{array}$ & \multicolumn{1}{c}{ Gameplay } \\
\hline Decomposição & $\begin{array}{l}\text { Para movimentar-se pela tela, o usuário precisa decompor, esquadrinhar a tela } \\
\text { em busca de saídas para cumprir os seus objetivos, seja fugir dos vilões ou levar } \\
\text { o lixo à lixeira }\end{array}$ \\
\hline Reconhecimento de \\
padrões & $\begin{array}{l}\text { Após o processo de separar por quadros a tela, o usuário começa ambientar-se } \\
\text { com os controles e os padrões de movimento e disposição dos obstáculos } \\
\text { definindo a existência de barreiras móveis e fixas a fim de montar estratégias de } \\
\text { movimento }\end{array}$ \\
\hline Abstração & $\begin{array}{l}\text { No processo de busca por objetivos, o usuário deve perceber que existem } \\
\text { possibilidades específicas para cumprir os dois objetivos gerais: fuga dos vilões e } \\
\text { levar o lixo à lixeira. Caso seu movimento aprisione uma peça, não existirá mais } \\
\text { a possibilidade de finalizar a fase com pontos completos }\end{array}$ \\
\hline Algoritmo & $\begin{array}{l}\text { O processo de traçar a melhor rota, sem perder vidas e cumprindo as metas } \\
\text { estabelecidas da coleta. }\end{array}$ \\
\hline
\end{tabular}

Tabela 4. Pensamento Computacional e o passo a passo do jogo. 


\section{Resultados e Discussões}

O propósito do desenvolvimento do jogo EcoLogic é o de fornecer uma ferramenta multidisciplinar, lúdica para a promoção do desenvolvimento do Pensamento Computacional de maneira formal e não formal, ou seja, o jogo pode tanto ser jogado de forma independente por qualquer usuário, como pode ser usado em sala de aula de forma colaborativa com a presença das crianças e seu tutor.

A proposta de desenvolvimento do jogo passou por alguns processos de avaliação para validar o produto durante suas etapas de construção sendo elas o design de aprendizagem e a análise temática. Nesse processo foram realizadas entrevistas com professores na área de Designer Instrucional e Educação Ambiental. As observações dos profissionais foram levadas em consideração nas etapas de discussão e proposta de arquitetura do jogo.

Mesmo o jogo tendo a temática voltada para Educação Ambiental, foi possível implementar mecânicas fundamentadas nos quatro pilares do Pensamento Computacional. O processo de criação seguiu cinco passos: i) Brainstorm, ii) Especificação dos Requisitos do Jogo, iii) Planejamento, iv) Prototipação e v) Testes.

Este trabalho explora mais fortemente a fase de Especificação dos Requisitos do Jogo. Nesta fase as mecânicas e processos do jogo foram mapeados e discutidos. Para que o processo de resolução dos problemas fosse apresentado em forma de missões no jogo, as mecânicas e processos foram ordenados de maneira a adequarem-se aos quatro pilares do pensamento computacional.

Foi realizada a avaliação quanto a aplicação da mecânica do mapa e do mundo do jogo para verificar a possibilidade de utilizar os quatro pilares do Pensamento Computacional. Na análise concluiu-se que os pilares defendidos como importantes para o desenvolvimento do Pensamento Computacional estão diluídos nas estruturas do jogo. A arquitetura tem a finalidade de engajar o jogador tanto com a sequência de passos a ser realizada quanto com a gamificação em si. Esta relação está sendo apresentada na Tabela 5. É possível observar que existem elementos em EcoLogic que estão presentes em jogos comerciais da mesma categoria, bem como os objetivos de aprendizagem previamente delineados estão envoltos no universo do jogo.

\begin{tabular}{|c|c|c|c|}
\hline $\begin{array}{l}\text { Pensamento } \\
\text { Computacional }\end{array}$ & EcoLogic & $\begin{array}{c}\text { Estruturas } \\
\text { Computacionais }\end{array}$ & Meio Ambiente \\
\hline Decomposição & $\begin{array}{l}\text { Setorização das áreas de ação } \\
\text { Localização de objetivos } \\
\text { Classificação de lixeiras e } \\
\text { inimigo }\end{array}$ & $\begin{array}{l}\text { Variáveis } \\
\text { Repetições } \\
\text { Condicionais }\end{array}$ & $\begin{array}{l}\text { Relação entre o jogo e o } \\
\text { meio (pessoa, árvores, } \\
\text { lixeira, lixos) }\end{array}$ \\
\hline Padrão & $\begin{array}{l}\text { Organização espacial } \\
\text { Número de árvores } \\
\text { Formação de obstáculos } \\
\text { Localização de alvos }\end{array}$ & \multirow{3}{*}{$\begin{array}{l}\text { Disjunção } \\
\text { Conjunção } \\
\text { Condicionais } \\
\text { Menor Caminho } \\
\text { Repetições }\end{array}$} & $\begin{array}{l}\text { Localização de padrão } \\
\text { Correlato a diferentes } \\
\text { ambientes }\end{array}$ \\
\hline Abstração & $\begin{array}{l}\text { Posição dos lixos e lixeiras } \\
\text { Movimentação dos inimigos } \\
\text { HUD de feedback } \\
\text { Corações (vidas) }\end{array}$ & & $\begin{array}{l}\text { Descarte do lixo na } \\
\text { respectiva lixeira } \\
\text { Poluição do ambiente pelos } \\
\text { inimigos }\end{array}$ \\
\hline Algoritmo & $\begin{array}{l}\text { Sequência de passos para: } \\
\text { - Contornar obstáculos } \\
\text { - Cumprir metas } \\
\text { - Contornar inimigos }\end{array}$ & & $\begin{array}{l}\text { Como proceder para manter } \\
\text { o ambiente bem cuidado e } \\
\text { promover sustentabilidade } \\
\text { ambiental }\end{array}$ \\
\hline
\end{tabular}

Tabela 5. Avaliação da aplicação dos quatro pilares do Pensamento Computacional no universo de EcoLogic. 
A última etapa da avaliação realizada diz respeito ao jogo enquanto produto e como o mesmo foi recepcionado por conhecedores de jogos. O objetivo foi avaliar o produto como item de entretenimento e sua importância para processos de aprendizagem.

Para a avaliação foi considerado um conjunto de itens que, entre outros objetivos, visa analisar a capacidade do jogo em gerar imersão. Foi utilizado o método de avaliação de jogos educacionais EGameFlow (Sweetser \& Wyeth, 2005), em uma adaptação de Fu et. al. (2009) cuja função é mensurar a diversão, imersão e o prazer do jogador durante uma jornada com fundo educacional. São utilizadas heurísticas de usabilidade e experiência do usuário. Dada a importância da interação e avaliação do usuário com o jogo educacional, o método EGameFlow procura uma escala de satisfação entre estes (usuário-jogo educacional), podendo auxiliar o game designer a compreender os pontos fortes e fracos do jogo na perspectiva do jogador (Tsuda, Sanches, Ferreira, Otsuka, \& Beder, 2014).

Para o psicólogo Mihaly Csikszentmihalyi (Sweetser \& Wyeth, 2005), o estado de fluxo define uma experiência gratificante em que o indivíduo fica imerso em uma atividade qualquer, por vontade própria . O método E-Game Flow faz uso de 7 categorias de análise: concentração, desafio, controle, objetivos, Feedback, imersão e interação, como definido na Tabela 6.

Os testes foram realizados com estudantes de graduação em computação (Engenharia e Licenciatura) e contou com uma unidade amostral $N=07$, entretanto, o jogo foi desenvolvido para crianças com faixa etária entre 07 e 12 anos, o que pode apresentar uma ameaça a validade, pois depende do nível de leitura da criança para a compreensão do storytelling e mecânicas do jogo. EcoLogic apresentou as melhores porcentagens ( 5 e 6) nos critérios de clareza dos objetivos (56\%) e Feedback $(53,32 \%)$ conforme informado na Tabela 7. Durante o jogo, muitos usuários demonstraram dificuldade em organizar os quebra-cabeças apresentados de forma a criar as melhores estratégias para levar o lixo até as suas lixeiras, sobretudos nas sessões em que havia inimigos como sentinelas, o que aponta para a necessidade de redução no nível de dificuldade da primeira fase, para que esteja de acordo com o público alvo.

\begin{tabular}{ll}
\hline \multicolumn{1}{c}{ Critério } & \multicolumn{1}{c}{ Descrição } \\
\hline Concentração & Verifica o foco e a atenção do usuário, na resolução dos problemas no jogo. \\
\hline Desafio & O quanto o usuário se sente desafiado, sem uma pressão excessiva e nem gerar apatia. \\
\hline Controle & O quanto o usuário tem o controle sobre os elementos e objetivos do jogo. \\
\hline $\begin{array}{l}\text { Clareza nos } \\
\text { Objetivos }\end{array}$ & Os objetivos devem ser especificados, deixando claro aos usuários sua missão. \\
\hline Feedback & $\begin{array}{l}\text { São as informações que o jogador deve receber acerca do seu progresso e resultados } \\
\text { de suas ações. }\end{array}$ \\
\hline Imersão & $\begin{array}{l}\text { Quando os usuários se sentem no mundo do jogo, perdendo o senso de tempo e } \\
\text { consciência do que acontece à sua volta. }\end{array}$ \\
\hline Interação & $\begin{array}{l}\text { Quando existe a oportunidade de interação com outros jogadores, competições, salas } \\
\text { de chat online e etc. }\end{array}$ \\
\hline
\end{tabular}

Tabela 6. Definição dos critérios de avaliação do E-Game Flow.

Quando perguntados sobre o jogo, os usuários entrevistados indicaram gostar da proposta, mas apontaram para a necessidade de melhorias no design, e como o jogo está em processo de desenvolvimento, as observações serão incorporadas ao próximo protótipo para testes, um processo iterativo e incremental até que o jogo fique pronto para uso comum. 


\begin{tabular}{|c|c|c|c|c|c|c|c|}
\hline \multirow{2}{*}{ Critérios } & \multicolumn{7}{|c|}{ Pontuação atribuída } \\
\hline & $\mathbf{0}$ & 1 & 2 & 3 & 4 & 5 & 6 \\
\hline Concentração & $5 \%$ & $5 \%$ & $15 \%$ & $20 \%$ & $25 \%$ & $15 \%$ & $15 \%$ \\
\hline Desafios & $16 \%$ & $18 \%$ & $12 \%$ & $18 \%$ & $10 \%$ & $18 \%$ & $8 \%$ \\
\hline Autonomia & $8,8 \%$ & $24,4 \%$ & $4,4 \%$ & $15,5 \%$ & $8,8 \%$ & $17,7 \%$ & $20 \%$ \\
\hline Clareza dos objetivos & $0 \%$ & $0 \%$ & $4 \%$ & $16 \%$ & $24 \%$ & $16 \%$ & $40 \%$ \\
\hline Feedback & $6,6 \%$ & $6,66 \%$ & $13,3 \%$ & $13,3 \%$ & $6,6 \%$ & $16,6 \%$ & $36,6 \%$ \\
\hline Imersão & $8,5 \%$ & $37,1 \%$ & $5,7 \%$ & $14,2 \%$ & $20 \%$ & $5,71 \%$ & $8,5 \%$ \\
\hline Interação Social & $53,3 \%$ & $26,6 \%$ & $0 \%$ & $6,6 \%$ & $6,6 \%$ & $0 \%$ & $6,6 \%$ \\
\hline
\end{tabular}

Tabela 7. Avaliação do jogo usando E-Game-Flow.

O propósito do desenvolvimento do jogo EcoLogic é o de fornecer uma ferramenta multidisciplinar e lúdica para promoção do desenvolvimento do Pensamento Computacional de maneira formal e não formal. O jogo pode tanto ser jogado individual, por qualquer usuário, como pode ser usado em sala de aula de forma colaborativa com a presença de crianças e seu tutor.

Uma das possiblidades de trabalho em sala de aula é a utilização da narrativa para realizar a compreensão do texto. O professor pode pedir que as crianças criem redações sobre a jornada de Sofia. As histórias podem ser uma ferramenta para análise de Algoritmos presentes nas narrativas, bem como o processo de compreensão do texto pode prover a extração dos outros três pilares do Pensamento Computacional ( Decomposição, padrão e abstração) (Pires et al., 2018). Durante essa atividade, o tutor pode ainda explorar a narrativa a partir do cenário do jogo e exaltar as palavras chaves do tipo "Se" a personagem for pela direita "então", "Se" lixeira na localização "x".

O cenário de EcoLogic além de permitir traçar as menores rotas possíveis para se alcançar um alvo ou fugir dos vilões, pode-se explorar trabalhos com planos cartesianos, senso de lateralidade, entre outros. O jogo pode ser simulado em uma lousa digital interativa, possibilitando uma participação de um maior número de pessoas que poderão traçar estratégias de forma colaborativa em grupos, além do engajamento pela competitividade.

Diante das discussões da inserção do Pensamento Computacional como componente curricular nas escolas, se faz necessário o desenvolvimento de métodos, técnicas e ferramentas que possam ser utilizados em sala de aula, ou fora dela, sempre tendo como objetivo o desenvolvimento das estruturas cognitivas no que tange a racionalização de problemas.

\section{Considerações finais}

O Pensamento Computacional constitui uma poderosa ferramenta teórica para solução de problemas e tem sido cada vez mais investigado no meio científico e aplicado educacionalmente. $\mathrm{O}$ trabalho apresentado demonstrou como os quatro pilares do Pensamento Computacional (PC) foram aplicados à mecânica de um jogo de propósito sério, cujo tema é o Ambiente e Sustentabilidade, demonstrando, com isso, que, conforme a afirmação de Wing (2017), PC pode sim permear diversas áreas do conhecimento e não somente a Ciência da Computação.

Como trabalhos em andamento, estão sendo acrescentadas novas fases ao jogo e inseridos mais elementos de recompensa, como easter eggs e power up's, de tal forma a se criar um gameplay mais divertido e manter o jogador imerso. Outra mudança em processo de desenvolvimento é o design do jogo. A nova versão tem estilo "material design" pela popularidade de aplicativos semelhantes indicados para a faixa etária do jogo. 
Os playtests iniciais reforçam a afirmação da literatura de que jogos desenvolvidos sobre a perspectiva do Pensamento Computacional podem proporcionar aos usuários uma experiência dinâmica, motivadora e desafiadora e, quando atrelados a assuntos ou conhecimentos de cunho social, como a sustentabilidade, possibilitam que o tema seja visto de maneira mais lúdica, o que promove uma aprendizagem mais eficiente.

Análises realizadas durante a execução do projeto, levam a considerar os jogos como ferramentas cujas estruturas permitem explorar o processo de aprendizagem humano, como vem ocorrendo ao longo do tempo. O que leva a afirmação "o jogo é um espaço cujas missões, regras e objetivos requerem a aplicação dos quatro pilares do Pensamento Computacional". Assim, recomenda-se a utilização de jogos como ferramentas e estratégias de aprendizagem, seja no processo de gamificação de tarefas seja na criação de jogos de propósito sério. Ao utilizar como tema os componentes curriculares obrigatórios utilizando estratégias em que os pilares do Pensamento Computacional estão bem definidos, é possível engajar crianças em atividades lúdicas ao mesmo tempo em que desenvolvem o Pensamento Computacional de forma implícita.

\section{Referências}

Abt, C. C. (1987). Serious games: University press of America.

BBC. (2018). KS3 Bitesize Computer Science: Introduction to computational thinking. Retrieved 17/05/2019, 2018, from https://www.bbc.com/bitesize/guides/zp92mp3/revision/1

Berg Marklund, B. (2013). Games in formal educational settings: Obstacles for the development and use of learning games. University of Skövde.

Caillois, R. (2001). Man, play, and games (B. Mayer, Trans.). Estados Unidos da America: University of Illinois Press.

Calvacanti, C. (1995). Desenvolvimento e natureza: estudos para uma sociedade sustentável: Cortez; Fundação Joaquim Nabuco.

Christian, B., \& Griffiths, T. (2016). Algorithms to Live By: The Computer Science of Human Decisions: Henry Holt and Company.

Cormen, T., Leiserson, C., Rivest, R., \& Stein, C. (2001). Introduction to algorithms (2 ${ }^{\mathrm{a}}$ ed.). Cambridge, Massachusetts: MIT Press.

Crawford, C. (1981). The future of computer wargaming. Computer Gaming World, 1(1), 3-7.

Culberson, J. (1997). Sokoban is PSPACE-complete. Edmonton, Alberta, Canada: Department of Computer Science of The University of Alberta.

Dehaene, S. (2014). Consciousness and the Brain: Deciphering How the Brain Codes Our Thoughts: Penguin Publishing Group.

Dichev, C., \& Dicheva, D. (2017). Gamifying education: what is known, what is believed and what remains uncertain: a critical review. International journal of educational technology in higher education, 14(1), 9.

Fogel, R. W. (1994). Economic growth, population theory, and physiology: the bearing of long-term processes on the making of economic policy: National Bureau of Economic Research.

Fu, F.-L., Su, R.-C., \& Yu, S.-C. (2009). EGameFlow: A scale to measure learners' enjoyment of e-learning games. Computers \& Education, 52(1), 101-112.

Gee, J. P. (2003). What video games have to teach us about learning and literacy. Computers in Entertainment (CIE), 1(1), 20-20.

Gros, B. (2007). Digital games in education: The design of games-based learning environments. Journal of research on technology in education, 40(1), 23-38. 
Hamari, J., Shernoff, D., Rowe, E., Coller, B., Asbell-Clarke, J., \& Edwards, T. (2016). Challenging games help students learn: An empirical study on engagement, flow and immersion in game-based learning. Computers in Human Behavior, 54, 170179.

Hedin, B., Lundström, A., Westlund, M., \& Markström, E. (2017). The energy piggy bank-A serious game for energy conservation. Paper presented at the 2017 Sustainable Internet and ICT for Sustainability (SustainIT).

Honda, F., Pires, F., Melo, D., \& Faria, I. (2019). Pi-Pi-Sat: um jogo para execício e aprendizagem da língua Sateré Mawé. Paper presented at the Anais dos Workshops do Congresso Brasileiro de Informática na Educação.

Hsiao, H.-C. (2007). A brief review of digital games and learning. Paper presented at the Digital Game and Intelligent Toy Enhanced Learning, 2007. DIGITEL'07. The First IEEE International Workshop on.

Huizinga, J. (2014). Homo ludens: o jogo como elemento da cultura. São Paulo: Editora Perspectiva.

Jacobi, P. R. (2003). Educação ambiental, cidadania e sustentabilidade. Cadernos de pesquisa(118), 189-205.

Katsaliaki, K., \& Mustafee, N. (2012). A survey of serious games on sustainable development. Paper presented at the Proceedings of the Winter Simulation Conference.

Klopfer, E., Haas, J., Osterweil, S., \& Rosenheck, L. (2018). Resonant Games: Design Principles for Learning Games that Connect Hearts, Minds, and the Everyday: MIT Press.

Ludwig, W., \& Anscombe, G. (1953). Philosophical investigations. London, Basic Blackw.

Mack, S. K., Eric, Schwartz, J., Jessell, T., Siegelbaum, S., \& Hudspeth, A. J. (2013). Principles of Neural Science. EUA: McGraw-Hill Education.

Mayer, R. (2015). On the need for research evidence to guide the design of computer games for learning. 50(4), 349-353.

McFarlane, A., Sparrowhawk, A., \& Heald, Y. (2002). Report on the educational use of games: TEEM (Teachers evaluating educational multimedia), Cambridge.

Meadows, D., \& Randers, J. (2012). The limits to growth: the 30-year update: Routledge.

Melo, D., Silva, R., Duarte, C., \& Pires, F. (2018). Robô Euroi: Estratégias Matemáticas para desenvolver o Pensamento Computacional. Paper presented at the Anais dos Workshops do Congresso Brasileiro de Informática na Educação.

Mitchell, A., \& Savill-Smith, C. (2004). The use of computer and video games for learning: A review of the literature.

Pessoa, M., Alencar, L. F., Araújo, L., Melo, R., \& Pires, F. (2019). Looking for Pets: a game for the logical reasoning development. Paper presented at the 2019 IEEE Frontiers in Education Conference (FIE).

Pires, F., Duarte, J. C., Silva Pessoa , L., Pereira, K., Melo, R., \& de Freitas, R. (2018). Uma análise cognitiva entre a emergência de padrões em narrativas infantis $e$ elementos do pensamento computacional. Paper presented at the Brazilian Symposium on Computers in Education (Simpósio Brasileiro de Informática na Educação-SBIE).

Pires, F., Lima, F. M. M., Melo, R., Bernardo, J. R. S., \& de Freitas, R. (2019). Gamification and Engagement: Development of Computational Thinking and the Implications in Mathematical Learning. Paper presented at the 2019 IEEE 19th International Conference on Advanced Learning Technologies (ICALT). 
Plass, J. L., Homer, B. D., \& Kinzer, C. K. (2015). Foundations of game-based learning. Educational psychologist, 50(4), 258-283.

Qian, M., \& Clark, K. R. (2016). Game-based Learning and 21st century skills: A review of recent research. Computers in Human Behavior, 63, 50-58.

Resnick, M., \& Robinson, K. (2017). Lifelong Kindergarten: Cultivating Creativity Through Projects, Passion, Peers, and Play: MIT Press.

Rogers, S. (2014). Level Up! The guide to great video game design. United Kingdon: John Wiley \& Sons.

Sachs, I. (1989). Desarrollo sustentable, bio-industrialización descentralizada y nuevas configuraciones rural-urbanas. El caso de la India y el Brasil. Pensamiento Iberoamericano, 16, 235-256.

Salen, K., \& Zimmerman, E. (2003). Rules of play: Fundamentals of game design. MIT Press Cambridge.

Santos, A. D., Strada, F., \& Bottino, A. (2018). Approaching sustainability learning via digital serious games.

Sauvé, L. (2005). Educação Ambiental: possibilidades e limitações.

Savi, R., \& Ulbricht, V. R. J. R. (2008). Jogos digitais educacionais: benefícios e desafios. $6(1)$.

Siche, R., Agostinho, F., Ortega, E., \& Romeiro, A. (2007). Índices versus indicadores: precisões conceituais na discussão da sustentabilidade de países.

Smith, N., Sutcliffe, C., \& Sandvik, L. (2014). Code club: bringing programming to UK primary schools through scratch. Paper presented at the Proceedings of the 45th ACM technical symposium on Computer science education.

Suits, B. (1978). The Grasshopper: Games. Life and Utopia.

Sutiono, A. P., Purwarianti, A., \& Iida, H. (2014). A mathematical model of game refinement. Paper presented at the International Conference on Intelligent Technologies for Interactive Entertainment.

Sweetser, P., \& Wyeth, P. (2005). GameFlow: a model for evaluating player enjoyment in games. Computers in Entertainment (CIE), 3(3), 3-3.

Tsuda, M., Sanches, V. M., Ferreira, T. G., Otsuka, J. L., \& Beder, D. M. (2014). Análise de métodos de avaliação de jogos educacionais. Proceedings of XIII SBGames, 12-14.

Wang, T.-L., \& Tseng, Y.-F. (2014). An empirical study: Develop and evaluation a mobile serious game on environmental education. Paper presented at the 2014 9th International Conference on Computer Science \& Education.

Wing, J. (2006). Computational thinking. Communications of the ACM, 49(3), 33-35.

Wing, J. (2017). Computational thinking influence on research and education for all. Italian Journal of Educational Technology, 25(2), 7-14. 